\title{
Eine Ethik für Öffentliche Gesundheit?
}

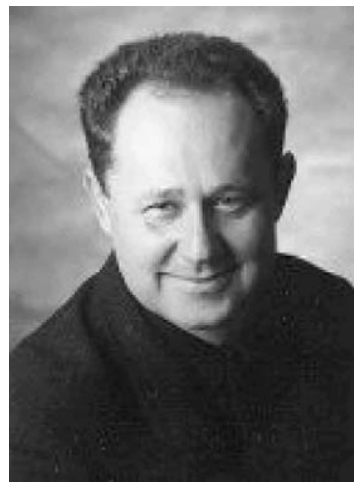

Prof. Dr. Manfred Wildner
Bibliografie

DOI http://dx.doi.org/

$10.1055 / \mathrm{s}-0031-1301333$

Gesundheitswesen 2012;

74: $1-2$

(c) Georg Thieme Verlag KG

Stuttgart · New York

ISSN 0941-3790

Korrespondenzadresse

Prof. Dr. M. Wildner

Bayerisches Landesamt für Gesundheit und Lebensmittelsicherheit

Veterinärstraße 2

85762 Oberschleißheim

manfred.wildner@Igl.bayern.de
„Der Herr sprach zu Mose: Befiehl den Israeliten, alle aus dem Lager hinauszuweisen, die an Aussatz oder Ausfluss leiden, auch jeden, der sich an einer Leiche verunreinigt" (Buch Numeri, 5. Kapitel, Verse 1 und 2). Die Anweisung zur Ausweisung ist eindeutig. Aus infektionshygienischer Sicht macht dieser Umgang mit vermutlich an übertragbaren Krankheiten Erkrankten („die an Aussatz oder Ausfluss leiden“) und auch Ansteckungsverdächtigen (,jeden, der sich an einer Leiche verunreinigt“) in erster Näherung Sinn. In einem modernen Verständnis wäre sicherlich u.a. die Sensitivität und Spezifität dieses Vorgehens kritisch zu hinterfragen, d.h., die Frage, wie häufig die falsch-positive oder auch falsch-negative Zuordnung von Erkrankten und Ansteckungsverdächtigen zu dieser doch recht drastischen Maßnahme des Infektionsschutzes ist. Und es würden sich im rechtsstaatlichen Raum auch die Kernfragen der Geeignetheit, Erforderlichkeit und Angemessenheit des Lagerverweises stellen. Der Grundsatz dieses Umganges mit Erkrankten und Ansteckungsverdächtigen, nämlich der einer Isolierung von der Gruppe der (noch) nicht Angesteckten bzw. Erkrankten, findet sich dabei weiter in unseren modernen Infektionsschutzgesetzen (siehe IfSG $\S \S 28-31$ ): Die dort gesetzlich benannten und bei Zuwiderhandlung mit Strafen bewehrten Schutzmaßnahmen umfassen Beobachtung (\$29), Quarantäne (§30) und berufliche Tätigkeitsverbote ( 331$)$.

Schutzmaßnahmen? Wohl eher nicht in Bezug auf die Erkrankten selbst. Hier ähneln die $\S \S 28-$ 31 IfSG sehr wohl den Versen 1 und 2 des 5. Buches Moses: Im Vordergrund steht der Schutz der Bevölkerung, im Konkreten der Schutz der Gesunden bzw. noch nicht Erkrankten. Zu allen Zeiten ist wohl davon auszugehen gewesen, dass die Hinausgewiesenen vermehrt Opfer der Unbill der Natur wie auch, als Randgruppe, der Zivilisation geworden sind und werden. Daran wird deutlich, was in unseren modernen Rechtparagrafen abgeschwächt immer noch präsent ist: das Spannungsverhältnis zwischen den Rechten des Individuums und den Schutzansprüchen der Gruppe. Dabei darf davon ausgegangen werden, dass nicht zuletzt durch die Sozialgesetzgebung inzwischen der Wohlfahrtsstaat des 20. und 21. Jahrhunderts seinen schützenden Mantel um die Erkrankten und ihre Familien legt, mehr als jemals zuvor. Und so diesen Kern menschlichen Lebens innerhalb der menschlichen Gemeinschaft schützt:„Das menschliche Leben stellt innerhalb der grundgesetzlichen Ordnung einen Höchstwert dar", urteilt das Bundesverfassungsgericht (BVerfGE 49,24 (53)).
Die staatlich bzw. gemeinschaftlich getragenen Maßnahmen betreffen Aspekte des Gesundheitsschutzes wie z.B. den Infektionsschutz, den umweltbezogenen Gesundheitsschutz oder auch Teile des Strafrechtes, sie betreffen Prävention und Gesundheitsförderung vom Arbeitsschutz bis zur Gestaltung gesundheitsförderlicher Lebenswelten in Schule und Stadtteilen, und sie umfassen die Regelwerke der gesundheitlichen Versorgung in den verschiedenen Sozialgesetzbüchern (SGB) wie dem SGB II zur Grundsicherung für Arbeitssuchende, SGB V zur gesetzlichen Krankenversicherung, SGB VI zur gesetzlichen Rentenversicherung, SGB VII zur gesetzlichern Unfallversicherung, SGB VIII zur Kinder- und Jugendhilfe, SGB IX zur Rehabilitation und Teilhabe behinderter Menschen, SGB XI zur Pflegeversicherung und SGB XII zur Sozialhilfe.

Moderne gesellschaftliche Ordnung: Anspruchsrechte in Form der 12 Sozialgesetzbücher anstelle der alttestamentarischen 10 Gebote, welche bei genauerem Hinsehen zumindest 8 Verbote sind? Das gesellschaftlich Gebotene, Sitte, Moral Ethik: Deutlich wird, dass ein Evolutionsprozess über 3 Jahrtausende hinsichtlich des sittlich Gebotenen im Umgang mit Gesundheit und Krankheit stattgefunden hat, der vom gewaltsamen Lagerausschluss alter Zeiten über den schützenden Mantel eines Heiligen Martin der christlicher Zeitenwende bis zu unseren heutigen umfangreichen sozialgesetzlichen Regelwerken geführt hat.

Um was für eine Art von Ethik handelt es sich? Ist es lediglich die Entfaltung der hippokratischen Berufsethik, welche die Kernethik des Gesundheitswesens ausmacht? Hier ist klar eine neue Komplexität und Pluralität der ethischen Bewertungen und Bewertungssysteme auszumachen, welche innerhalb des Gesundheitssystems zum Tragen kommen. Die ärztliche Berufsethik wie auch andere Berufsethiken nehmen weiterhin einen wichtigen Platz ein und haben die besondere Schutzbedürftigkeit von kranken Menschen wie auch das besondere Verhältnis der mit der Rolle des Begleitens, Linderns und Heilens Betrauten zu den Patienten und untereinander im Blick. Die ethischen Diskussionen werden bisweilen leidenschaftlich geführt - was gut so ist: schließlich wird um Werte und Schicksale gestritten. Oft sind existentielle Phasen des menschlichen Daseins betroffen, Anfang und das Ende des Lebens: Zeugung/Geburt und ein Sterben in Würde. Nicht zuletzt im Zusammenhang mit der sozialen Gesetzgebung und der Übernahme von gesamtgesellschaftlicher Verantwortung für den Schutz von Gesundheit und die Wiederherstellung und Behandlung bei Krankheit und ihrer Finanzierung 
sind neue ethische Aspekte hinzugekommen, vermehrt auch die Lebensabschnitte zwischen Geburt und Tod betreffend. Im „System Gesundheitswesen“ sehen wir uns so mit einer Vielfalt von ethischen Ansätzen konfrontiert: Neben der ärztlichen Berufsethik in der heutigen Form des Genfer Ärztegelöbnisses finden sich die ethische Positionen in Zusammenhang mit biomedizinischer Forschung z. B. in der Deklaration von Helsinki oder formuliert in den Prinzipien von Beauchamp und Childress (1994): Patienten- bzw. Probandenwohl, Schadensvermeidung, Wahrung der Patientenautonomie und Gerechtigkeit. Es gibt darüber hinaus vielfältige ethische Ansätze in Zusammenhang mit Verteilungsfragen und staatlicher Organisation. Gerade im letzten Bereich finden sich laufende Diskussionsprozesse, die z.B. die Form von Diskussionen um Rationalisierung, Rationierung und Priorisierung, um Solidarität und Eigenverantwortung, um personalisierte und wunscherfüllende Medizin annehmen können.

Typische Positionen in diesem ethischen Diskurs sind die Maximierung des Nutzens für die Bevölkerung insgesamt (Utilitarismus mit seinen Spielarten der gesundheitsökonomischen Evaluation), die Berufung auf Gebotenes (deontologische Standpunkte, häufig auf Basis von Berufsethik oder religiöser Überzeugung) und nicht zuletzt im sozialen Raum unseres Rechtsstaats die Rücksichtnahme auf gesetzte Rechte und Freiheiten (Liberalismus): „Die Würde des Menschen ist unantastbar. Sie zu achten und zu schützen ist Verpflichtung aller staatlichen Gewalt", heißt es in Artikel 1 des Grundgesetzes. Der Mensch also als Mitte und Maß. Im Bereich der öffentlich verantworteten Gesundheitsbelange sind dennoch die inhaltlichen Streitpunkte breit. Sie können Maßnahmen des Gesundheitsschutzes wie obligatorische Impfungen betreffen, Fragen der Zugangs- und Verteilungsgerechtigkeit im Rahmen der gesetzlichen Krankenversorgung und auch Fragen der optimalen Ressourcenallokation von Seiten der Financiers des Gesamtsystems. Für den erstgenannten Bereich des staatlich verantworteten Gesundheitsschutzes ist ein abgestuftes Vorgehen des Handelns skizziert worden, welches z.B. für Impfungen von der Erlaubnis über Empfehlungen und Kostenübernahmen bis zur Impfpflicht führen kann (Nuffield Council on Bioethics 2007, Marckmann 2008). Dem gegenüber findet sich insbesondere im letztgenannten Bereich häufig ein zumindest rhetorisches Primat des utilitaristischen Standpunktes: der Argumente von Effizienz und Kosten-Nutzen-Kalkülen, effektiver Ressourcenallokationen und wirtschaftlicher Rationalität. Der Patient als Kunde? Der ethische Diskurs ist in diesen Punkten noch lange nicht am Ende. Im Bereich von Öffentlicher Gesundheitspflege/ Public Health sind auch schon Grundlagen für eine diesbezügliche Diskussion gelegt worden, auch vor dem Hintergrund der Herausforderungen und Hoffnungen der Genomik (Brand et al., 2008, Strech und Marckmann 2010, Kuhn und Wildner 2011).

„Ärztinnen und Ärzte dienen der Gesundheit des einzelnen Menschen und der Bevölkerung“, lautet Satz 1 in Artikel 1 der Ärztlichen Berufsordnung. Dieser kurze Satz schlägt einen erheblichen Spannungsbogen mit vielfältigen potentiellen Konflikten im Verhältnis zwischen der Gesundheit des Einzelnen und der Gesundheit der Bevölkerung. In unserem modernen Gesundheitssystem betrifft der ethische Konflikt nur mehr selten Quarantäne und Absonderung (negative Abwehrrechte), häufig jedoch die Gewährung oder Versagung von Leistungen, die möglich, vielleicht wünschenswert sind, jedoch nicht ohne weiteres von der Solidargemeinschaft getragen werden (positive Anspruchsrechte). Rehabilitationsaufenthalte, Implantate, Medikamente, ärztliche Sprechzeit - unser medizinisches „Lagerleben“ hat oft nur kurze Pausen, bevor wieder aufgebrochen werden muss: vom kurzen Termin beim niedergelassenen Arzt zur getakteten Zeit bei der Krankengymnastik, vom DRG-geregelten Krankenhausaufenthalt zur auf Evidenz und Effizienz getrimmten Rehabilitationsmaßnahme. Die kurzen Pausen gelten auch für die Leistungserbringer. Nicht eine Ethik, sondern viele Ethiken melden sich dabei zu Wort.

Derartige ethische Fragen kommen in der einen oder anderen Form in den Beiträgen dieses Hefts zum Tragen: Patientenbeschwerden beim Bundespatientenbeauftragten, Niederlassungsentscheidungen künftiger Hausärzte, Patienten als Kunden, dem Wettbewerb zwischen Generika und Originalherstellern in Österreich, Angst und Depression bei Älteren, Stillförderung, zahnärztlichen Gutachten und der Priorisierung in der medizinischen Versorgung in Norwegen.

Ein klares „Ja“ zur Pluralität ethischer Positionen und Herangehensweisen in den vielfältigen Themen des Gesundheitswesens - es darf und muss gestritten werden. Hat damit die Diskursethik das letzte Wort? Bei aller Notwendigkeit des ethischen Diskurses scheint eine Unterscheidung zwischen den unterschiedlichen ethischen Ansätzen wichtig: Erst wenn der Kern der menschlichen Würde und (Menschen-)Rechte geschützt ist, können sich die weiteren Diskussionen gleichsam in den Falten dieses schützenden Mantels entwickeln (Wildner et al., 2010).

A propos Mantel: Als Martin von Tours im Jahre des Herrn 334 als Reiter der kaiserlichen Garde im gallischen Amiens stationiert war, verfügte er über den typischen weißen, im oberen Bereich mit Schaffell gefütterten Mantel: Der Rock des Kaisers ist zwar eng, doch er wärmt. Der Legende nach soll er an einem kalten Wintertag einem armen, unbekleideten Mann draußen vor den (Stadt-)Toren von Amiens die Hälfte seines Militärmantels zugeworfen haben, was einen mutigen Schnitt des Teilens mit dem Schwert voraussetzte. Damit wurde vorweggenommen, was moderne Rechtsprechungen bzw. ein moderner Rechtsstaat auch von der Gemeinschaft fordern: den unbedingten Schutz eines Kerns an unveräußerlichen Werten und Menschenwürde. Dies kann Einschnitte und Teilen bedeuten. Dass der Schutz der elementaren und unverzichtbaren Rechte einzelner Menschen nicht zur Disposition gestellt werden darf, auch bei den ganz alltäglichen Entscheidungen in der Patientenversorgung, siehe die Beiträge in diesem Heft, und dass dieser Vorbehalt einer notwendigen Diskussion auch einmal vorangestellt werden darf, ist eine wichtige Botschaft dieser Geschichte.

\section{Literatur}

1 Beauchamp TL, Childress JF. Principles of Biomedical Ethics. Oxford: Oxford University Press; 1994, 2001

2 Borchert $W$. Draußen vor der Tür. Ein Stück, das kein Theater spielen und kein Publikum sehen will. Hamburg: Rowohlt Verlag; 1947 (Erstausgabe)

3 Brand A, Brand H, Schulte in den Bäumen T. The impact of genetics and genomics on public health. European Journal of Human Genetics 2008; 16: 5-13

4 Kuhn J, Wildner M. Ethik in der Gesundheitsförderung und Prävention. In: Blümel S, Franzkowiak P, Kaba-Schönstein L, Nöcker G, Trojan A, Hrsg. Leitbegriffe der Gesundheitsförderung und Prävention. Köln: Bundeszentrale für gesundheitliche Aufklärung 2011; 65-68

5 Marckmann G. Impfprogramme im Spannungsfeld zwischen individueller Autonomie und allgemeinem Wohl. Bundesgesundheitsbl Gesundheitsforsch - Gesundheitsschutz 2008; 51: 175-183

6 Nuffield Council on Bioethics. Public Health: Ethical issues. Cambridge: Cambridge Publ; 2007; 47

7 Strech D, Marckmann G. Public Health Ethik. Berlin: LIT Verlag; 2010

8 Wildner $M$, Zöllner $H$, Zapf A. Eine Ethik für öffentliche Gesundheit. In: Wildner M, Günther S, Kerscher G, Zapf A, Hrsg. Brücken bauen: Die Rolle des Arztes und seine Tätigkeit in einem modernen ÖGD. Symposium am 22. bis 24. Juli 2009 in Wildbad Kreuth Germering: Zuckschwerdt Verlag; 2010; 86-99 